\title{
The new integrable deformations of short pulse equation and sine-Gordon equation, and their solutions
}

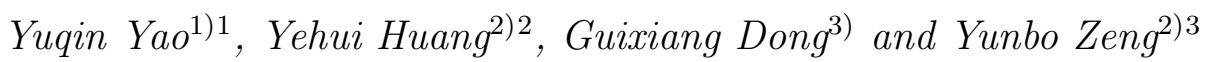 \\ 1) Department of Applied Mathematics, China Agricultural University, Beijing, 100083, PR \\ China \\ 2) Department of Mathematical Science, Tsinghua University, Beijing, 100084, PR China \\ 3) College of Science,Shandong Jianzhu University, Shandong Jinan 250101 PR China
}

\begin{abstract}
We first derive an integrable deformed hierarchy of short pulse equation and their Lax representation. Then we concentrated on the solution of integrable deformed short pulse equation (IDSPE). By proposing a generalized reciprocal transformation, we find a new integrable deformed sine-Gordon equation (IDSGE) and its Lax representation. The multisoliton solutions, negaton solutions and positon solutions for the IDSGE and the N-loop soliton solutions, N- negaton and N-positon solutions for the IDSPE are presented. In the reduced case the new N-positon solutions and N-negaton solutions for short pulse equation are obtained.
\end{abstract}

Key words: integrable deformed short pulse equation, integrable deformed sineGordon equation, reciprocal transformation, loop soliton solution, soliton solution

\section{Introduction}

It is known that pulse propagation in optical fibers is usually modeled by the cubic nonlinear Schrödinger equaion [1]. However, it is not valid for the ultra-short pules. In 2004, starting from the Maxwell equation of electric field in the fiber, Schäfer and Wayne derived the short pulse equation(SPE) [2]

$$
u_{x t}=u+\frac{1}{6}\left(u^{3}\right)_{x x}
$$

as an alternative of the cubic nonlinear Schrödinger equation to describing the propagation of ultrashort optical pules in nonlinear media, where $u(x, t)$ represents the magnitude of electric field. In recent years, the short pulse model has attracted considerable attention. In [3,4], it was also deduced from the partial differential equation which describes pseudospherical surfaces. In [5], Sakovich and Sakovich proved that the SPE is integrable by discovering a Lax pair for the SPE. Brunelli proved the integrability of the SPE from the Hamiltonian point of view and studied the short pulse hierarchy in [6,7]. In [8], Feng

\footnotetext{
${ }^{1}$ Corresponding author: yyqinw@126.com

${ }^{2}$ huangyh@mails.tsinghua.edu.cn

3 yzeng@math.tsinghua.edu.cn
} 
et al. proposed the integrable semi-discrete and full-discrete analogues of the SPE. As far as the solutions of the SPE are considered, in [9] not only solitary wave solutions are obtained by making use of transformation between the SPE and sine-Gordon equation but also the pulse solutions of the SPE were derived from the breather solutions of the SG equation. Some periodic and traveling wave solutions of SPE are given in [10]. Kuetche et al. constructed the two loop soliton solutions with use of bilinear method and hodograph transformation [11]. In [12,13], Matsuno develop a systematic procedure to construct the periodic solutions and multiloop solitons.

The integrable deformation of integrable system attract a lot of interests from both physical and mathematical points. One kind of the integrable deformation is the socalled soliton equation with self-consistent sources [14]- [21], which consists of the soliton equation with additional terms by coupling the corresponding eigenvalue problems, and has important application. For example, the nonlinear Schrödinger equation with selfconsistent sources is relevant to some problems of plasma physical and solid state physics.

In this paper, we consider the integrable deformed short pulse equation (IDSPE) which has not been studied yet. First we derive the integrable deformed hierarchy of short pulse equation(IDSPH) and their Lax representation, which includes integrable deformed short pulse equation (IDSPE) and its Lax representation. This implies that the IDSPE is Lax integrable. A generalized reciprocal transformation for the IDSPE is proposed. This transformation converts the IDSPE and its Lax representation into new integrable deformed sine-Gordon equation (IDSGE) and its Lax representation. The IDSGE can be written as bilinear form by introducing an independent variable transformation. The $\mathrm{N}$-soliton solutions of the sine-Gordon equation were obtained in [22,23], and the positon solutions of sine-Gordon equation and its properties were studied in [23]. Here, we find the N-soliton solutions, N-negatons solutions and N-positon solutions for the IDSGE . Further using the inverse reciprocal transformation, we construct N-loop soliton solutions, N-negaton solutions and N-positon solutions of the IDSPE. In the reduced case we obtain the new N-negaton solutions and new N-positon solutions for the short pulse equation.

This paper is organized as follows. In section 2, we establish the IDSPH and its Lax representation. In section 3, A generalized reciprocal transformation for the IDSPE is proposed and the IDSGE is worked out. In section 4, the solutions of the IDSGE are obtained. Section 5 gives the solutions of IDSPE. The conclusion is given in section 6 .

\section{The new integrable deformed hierarchy of short pulse equation (IDSPH) and its Lax pair}

\subsection{The IDSPH}

Consider the eigenvalue problem [5]

$$
\left(\begin{array}{l}
\varphi_{1} \\
\varphi_{2}
\end{array}\right)_{x}=U\left(\begin{array}{c}
\varphi_{1} \\
\varphi_{2}
\end{array}\right), \quad U=\left(\begin{array}{cc}
\lambda & \lambda u_{x} \\
\lambda u_{x} & -\lambda
\end{array}\right) .
$$


The adjoint representation reads

$$
V_{x}=[U, V]
$$

Set

$$
V=\left(\begin{array}{cc}
A & B \\
C & -A
\end{array}\right)=\sum_{m=0}^{\infty}\left(\begin{array}{cc}
\lambda a_{m} & \lambda u_{x} a_{m}+b_{m} \\
\lambda u_{x} a_{m}+c_{m} & -\lambda a_{m}
\end{array}\right) \lambda^{m} .
$$

Eq. (3) yields

$$
\left\{\begin{array}{l}
a_{m, x}=u_{x}\left(c_{m}-b_{m}\right), \\
b_{m+1, x}=2 b_{m}-\left(u_{x} a_{m}\right)_{x}, \\
c_{m+1, x}=-2 c_{m}-\left(u_{x} a_{m}\right)_{x} .
\end{array}\right.
$$

Taking $b_{0}=c_{0}=0, a_{0}=\frac{1}{4}$, we have

$$
\left\{\begin{array}{c}
b_{1}=c_{1}=-\frac{1}{4} u_{x}, a_{1}=0, \\
b_{2}=-c_{2}=-\frac{1}{2} u, a_{2}=\frac{1}{2} u^{2}, \cdots,
\end{array}\right.
$$

and in general,

$$
\begin{aligned}
& b_{2 n}=-c_{2 n}=2 \partial^{-1} b_{2 n-1}, \\
& a_{2 n}=-2 \partial^{-1}\left(u_{x} b_{2 n}\right), a_{2 n+1}=0, \\
& b_{2 n+1}=c_{2 n+1}=2 \partial^{-1} b_{2 n}-u_{x} a_{2 n}=L b_{2 n-1}, L=4\left(\partial^{-1}+u_{x} \partial^{-1} u_{x}\right) \partial^{-1},
\end{aligned}
$$

where $\partial=\frac{\partial}{\partial x}, \partial \partial^{-1}=\partial^{-1} \partial=1$.

Set

$$
V^{(n)}=\sum_{m=0}^{2 n}\left(\begin{array}{cc}
\lambda a_{m} & \lambda u_{x} a_{m}+b_{m} \\
\lambda u_{x} a_{m}+c_{m} & -\lambda a_{m}
\end{array}\right) \lambda^{m-2 n}
$$

and take

$$
\left(\begin{array}{l}
\varphi_{1} \\
\varphi_{2}
\end{array}\right)_{t_{n}}=V^{(n)}\left(\begin{array}{l}
\varphi_{1} \\
\varphi_{2}
\end{array}\right)
$$

Then the compatibility condition of Eqs.(21) and (9) gives rise to the short pulse hierarchy $(\mathrm{SPH})$

$$
u_{x t_{n}}=-\partial b_{2 n+1}, n=0,1, \cdots .
$$

When $n=1$, (10) gives the short pulse equation

$$
u_{x t}=u+\frac{1}{6}\left(u^{3}\right)_{x x}
$$

and $V^{(2)}$ in (9) is given by

$$
V^{(2)}=\left(\begin{array}{cc}
\frac{1}{2} \lambda u^{2}+\frac{1}{4 \lambda} & \frac{1}{2} \lambda u^{2} u_{x}-\frac{u}{2} \\
\frac{1}{2} \lambda u^{2} u_{x}+\frac{u}{2} & -\frac{1}{2} \lambda u^{2}-\frac{1}{4 \lambda}
\end{array}\right) .
$$


For $n$ distinct real $\lambda_{j}$, consider the following spectral problem

$$
\left(\begin{array}{c}
\varphi_{1 j} \\
\varphi_{2 j}
\end{array}\right)_{x}=\left(\begin{array}{cc}
\lambda_{j} & \lambda_{j} u_{x} \\
\lambda_{j} u_{x} & -\lambda_{j}
\end{array}\right)\left(\begin{array}{c}
\varphi_{1 j} \\
\varphi_{2 j}
\end{array}\right) .
$$

It is easy to find that

$$
\frac{\delta \lambda_{j}}{\delta u}=-2 \lambda_{j}\left(\varphi_{1 j}^{2}+\varphi_{2 j}^{2}\right), L\left(\varphi_{1 j}^{2}+\varphi_{2 j}^{2}\right)_{x}=\frac{1}{\lambda_{j}^{2}}\left(\varphi_{1 j}^{2}+\varphi_{2 j}^{2}\right)_{x}
$$

According to the approach proposed in Refs. [17]- [21], the short pulse hierarchy with self-consistent sources (SPHSCS) or integrable deformed short pulse hierarchy (IDSPH) is defined by

$$
\begin{aligned}
& u_{x t_{n}}=-\partial\left[b_{2 n+1}-\sum_{j=1}^{N} \frac{1}{2 \lambda_{j}^{2}}\left(\varphi_{1 j}^{2}+\varphi_{2 j}^{2}\right)_{x}\right], \\
& \varphi_{1 j x}=\lambda_{j} \varphi_{1 j}+\lambda_{j} u_{x} \varphi_{2 j}, \varphi_{2 j x}=\lambda_{j} u_{x} \varphi_{1 j}-\lambda_{j} \varphi_{2 j}, j=1,2, \cdots, N .
\end{aligned}
$$

When $n=1$, (14) gives the short pulse equation with self-consistent sources (SPESCS) or integrable deformed short pulse equation (IDSPE)

$$
\begin{aligned}
& u_{x t}=u+\frac{1}{6}\left(u^{3}\right)_{x x}+\sum_{j=1}^{N} \frac{1}{2 \lambda_{j}^{2}}\left(\varphi_{1 j}^{2}+\varphi_{2 j}^{2}\right)_{x x}, \\
& \varphi_{1 j x}=\lambda_{j} \varphi_{1 j}+\lambda_{j} u_{x} \varphi_{2 j}, \varphi_{2 j x}=\lambda_{j} u_{x} \varphi_{1 j}-\lambda_{j} \varphi_{2 j}, j=1,2, \cdots, N .
\end{aligned}
$$

\subsection{Lax pair of the IDSPH}

In order to find the Lax pair for IDSPE (15), we first consider the following stationary equation of (15).

$$
\begin{aligned}
& b_{3}-\sum_{j=1}^{N} \frac{1}{2 \lambda_{j}^{2}}\left(\varphi_{1 j}^{2}+\varphi_{2 j}^{2}\right)_{x}=0, \\
& \varphi_{1 j x}=\lambda_{j} \varphi_{1 j}+\lambda_{j} u_{x} \varphi_{2 j}, \varphi_{2 j x}=\lambda_{j} u_{x} \varphi_{1 j}-\lambda_{j} \varphi_{2 j}, j=1,2, \cdots, N .
\end{aligned}
$$

According to Eqs.(6), (17), (13) and (16), we may define

$$
\begin{aligned}
& \bar{a}_{0}=\frac{1}{4}, \bar{b}_{0}=\bar{c}_{0}=0, \bar{b}_{1}=\bar{c}_{1}=-\frac{1}{4} u_{x}, \bar{a}_{1}=0, \bar{b}_{2}=-\bar{c}_{2}=-\frac{1}{2} u, \bar{a}_{2}=\frac{1}{2} u^{2}, \\
& \bar{b}_{2 n+1}=\bar{c}_{2 n+1}=L^{n-1} \bar{b}_{3}=L^{n-1} \sum_{j=1}^{N} \frac{1}{2 \lambda_{j}^{2}}\left(\varphi_{1 j}^{2}+\varphi_{2 j}^{2}\right)_{x}=\sum_{j=1}^{N} \frac{1}{2 \lambda_{j}^{2 n}}\left(\varphi_{1 j}^{2}+\varphi_{2 j}^{2}\right)_{x}, n=1,2, \cdots, \\
& \bar{b}_{2 n}=-\bar{c}_{2 n}=2 \partial^{-1} \bar{b}_{2 n-1}=\sum_{j=1}^{N} \frac{1}{\lambda_{j}^{2 n-2}}\left(\varphi_{1 j}^{2}+\varphi_{2 j}^{2}\right), n=2,3, \cdots,
\end{aligned}
$$




$$
\begin{aligned}
& \bar{a}_{2 n}=-2 \partial^{-1}\left(u_{x} \bar{b}_{2 n}\right)=-2 \partial^{-1} \sum_{j=1}^{N} \frac{1}{\lambda_{j}^{2 n-2}} u_{x}\left(\varphi_{1 j}^{2}+\varphi_{2 j}^{2}\right)=-2 \sum_{j=1}^{N} \frac{1}{\lambda_{j}^{2 n-1}} \varphi_{1 j} \varphi_{2 j}, \quad n=2,3, \cdots \\
& \bar{a}_{2 n+1}=0, \quad n=1,2,3, \cdots
\end{aligned}
$$

Then we have

$$
\begin{gathered}
\bar{A}=\lambda^{-2} \sum_{n=0}^{\infty} \bar{a}_{n} \lambda^{n+1}=\frac{1}{4 \lambda}+\frac{1}{2} u^{2}+\bar{A}_{0}, \\
\bar{A}_{0}=\sum_{n=2}^{\infty} \bar{a}_{2 n} \lambda^{2 n-1}=-2 \sum_{j=1}^{N} \sum_{n=2}^{\infty}\left(\frac{\lambda}{\lambda_{j}}\right)^{2 n-1} \varphi_{1 j} \varphi_{2 j}=2 \lambda \sum_{j=1}^{N} \frac{1}{\lambda_{j}} \varphi_{1 j} \varphi_{2 j}+2 \lambda \sum_{j=1}^{N} \frac{\lambda_{j}}{\lambda^{2}-\lambda_{j}^{2}} \varphi_{1 j} \varphi_{2 j} .
\end{gathered}
$$

In the same way, we find that

$$
\begin{gathered}
\bar{V}=\left(\begin{array}{cc}
\bar{A} & \bar{B} \\
\bar{C} & -\bar{A}
\end{array}\right)=\lambda^{-2} \sum_{n=0}^{\infty}\left(\begin{array}{cc}
\lambda \bar{a}_{n} & \lambda u_{x} \bar{a}_{n}+\bar{b}_{n} \\
\lambda u_{x} \bar{a}_{n}+\bar{c}_{n} & -\lambda \bar{a}_{n}
\end{array}\right) \lambda^{n} \\
=V^{(2)}+N_{0}, \quad N_{0}=\left(\begin{array}{cc}
\bar{A}_{0} & \bar{B}_{0} \\
\bar{C}_{0} & -\bar{A}_{0}
\end{array}\right) \cdot \\
\bar{B}_{0}=\sum_{j=1}^{N}\left[\frac{2 \lambda}{\lambda_{j}} u_{x} \varphi_{1 j} \varphi_{2 j}-\left(\varphi_{1 j}^{2}+\varphi_{2 j}^{2}\right)-\frac{\lambda_{j}}{\lambda}\left(\varphi_{1 j}^{2}-\varphi_{2 j}^{2}\right)+\frac{\lambda_{j}^{2}}{\lambda^{2}-\lambda_{j}^{2}}\left[\frac{\lambda_{j}}{\lambda}\left(\varphi_{2 j}^{2}-\varphi_{1 j}^{2}\right)-\left(\varphi_{1 j}^{2}+\varphi_{2 j}^{2}\right)\right]\right] . \\
\bar{C}_{0}=\sum_{j=1}^{N}\left[\frac{2 \lambda}{\lambda_{j}} u_{x} \varphi_{1 j} \varphi_{2 j}+\left(\varphi_{1 j}^{2}+\varphi_{2 j}^{2}\right)-\frac{\lambda_{j}}{\lambda}\left(\varphi_{1 j}^{2}-\varphi_{2 j}^{2}\right)+\frac{\lambda_{j}^{2}}{\lambda^{2}-\lambda_{j}^{2}}\left[\frac{\lambda_{j}}{\lambda}\left(\varphi_{2 j}^{2}-\varphi_{1 j}^{2}\right)+\left(\varphi_{1 j}^{2}+\varphi_{2 j}^{2}\right)\right]\right] .
\end{gathered}
$$

Since $\bar{a}_{n}, \bar{b}_{n}$ and $\bar{c}_{n}$ satisfy the same recursion relations as (5) . It is obvious that $\bar{V}$ satisfies

$$
\bar{V}_{x}=[U, \bar{V}] .
$$

In fact, it is easy to verify that (18) under (16b) leads to (16a). Since (16) is the stationary equation of (15), we immediately obtain the zero curvature representation for IDSPE (15)

$$
U_{t}-\bar{V}_{x}+[U, \bar{V}]=0
$$

with the Lax pair for the IDSPE (15)

$$
\begin{aligned}
& \left(\begin{array}{l}
\phi_{1} \\
\phi_{2}
\end{array}\right)_{x}=\left(\begin{array}{cc}
\lambda & \lambda u_{x} \\
\lambda u_{x} & -\lambda
\end{array}\right)\left(\begin{array}{c}
\phi_{1} \\
\phi_{2}
\end{array}\right), \\
& \left(\begin{array}{c}
\phi_{1} \\
\phi_{2}
\end{array}\right)_{t}=\bar{V}\left(\begin{array}{c}
\phi_{1} \\
\phi_{2}
\end{array}\right) .
\end{aligned}
$$

Furthermore, the zero curvature representation and Lax pair for IDSPH (14) are given by (19) and (20) with

$$
\bar{V}=V^{(n)}+N_{0}
$$

Remark 1. The zero curvature representation and Lax pair for IDSPH (14) are given by (19), (20) and (21). This implies that the new IDSPH is integrable in the Lax sense. 


\section{The new integrable deformed sine-Gordon equa- tion (IDSGE)}

By introducing the new dependent variable [12]

$$
r^{2}=1+u_{x}^{2}
$$

Eq. (15a) is transformed into the form

$$
r_{t}=\frac{1}{2}\left(u^{2} r\right)_{x}+\frac{u_{x}}{2 r} \sum_{j=1}^{N} \lambda_{j}^{-2}\left(\varphi_{1 j}^{2}+\varphi_{2 j}^{2}\right)_{x x}=\left(\frac{1}{2} u^{2} r+2 r \sum_{j=1}^{N} \lambda_{j}^{-1} \varphi_{1 j} \varphi_{2 j}\right)_{x} .
$$

So we can define a reciprocal transformation $(x, t) \rightarrow(y, s)$ by the relation

$$
d y=r d x+\left(\frac{1}{2} u^{2} r+2 r \sum_{j=1}^{N} \lambda_{j}^{-1} \varphi_{1 j} \varphi_{2 j}\right) d s, \quad d s=d t
$$

and we have

$$
\frac{\partial}{\partial x}=r \frac{\partial}{\partial y}, \quad \frac{\partial}{\partial t}=\frac{\partial}{\partial s}+\left(\frac{1}{2} u^{2} r+2 r \sum_{j=1}^{N} \lambda_{j}^{-1} \varphi_{1 j} \varphi_{2 j}\right) \frac{\partial}{\partial y}
$$

Denoting $\phi_{i}(x, t)=\psi_{i}(y, s), \varphi_{i j}(x, t)=\psi_{i j}(y, s),(i=1,2)$, with the new variable $y$ and $s,(22)$ and (23) are transformed into

$$
\begin{gathered}
r^{2}=1+r^{2} u_{y}^{2} \\
r_{s}=r^{2} u u_{y}+2 r^{2} \sum_{j=1}^{N} \lambda_{j}^{-1}\left(\psi_{1 j} \psi_{2 j}\right)_{y}
\end{gathered}
$$

respectively. Furthermore, we define

$$
u_{y}=\sin z, z=z(y, s) .
$$

Inserting (28) into (26) gives rise to

$$
r=\frac{1}{\cos z}
$$

Using (25) and (29), (15b) is converted the following form

$$
\psi_{1 j y}=\lambda_{j} \cos z \psi_{1 j}+\lambda_{j} \sin z \psi_{2 j}, \psi_{2 j y}=\lambda_{j} \sin z \psi_{1 j}-\lambda_{j} \cos z \psi_{2 j}, j=1,2, \cdots, N .
$$

Under Eqs.(28)-(30), (27) becomes

$$
z_{s}=u+2 \sum_{j=1}^{N}\left(\psi_{1 j}^{2}+\psi_{2 j}^{2}\right)
$$


So under the reciprocal transformation (25), the IDSPE (15) is transformed into the following integrable deformed sine-Gordon equation (IDSGE)

$$
\begin{aligned}
& z_{y s}=\sin z+2 \sum_{j=1}^{N}\left(\psi_{1 j}^{2}+\psi_{2 j}^{2}\right)_{y}, \\
& \psi_{1 j y}=\lambda_{j} \cos z \psi_{1 j}+\lambda_{j} \sin z \psi_{2 j}, \psi_{2 j y}=\lambda_{j} \sin z \psi_{1 j}-\lambda_{j} \cos z \psi_{2 j}, j=1,2, \cdots, N .
\end{aligned}
$$

Under the reciprocal transformation (25), (28), (29) and (31), the Lax pair (20) for the IDSPE (15) are transformed into the Lax pair for (32)

$$
\begin{gathered}
\left(\begin{array}{l}
\psi_{1} \\
\psi_{2}
\end{array}\right)_{y}=\left(\begin{array}{cc}
\lambda \cos z & \lambda \operatorname{sinz} \\
\lambda \sin z & -\lambda \cos z
\end{array}\right)\left(\begin{array}{c}
\psi_{1} \\
\psi_{2}
\end{array}\right) \\
\left(\begin{array}{l}
\psi_{1} \\
\psi_{2}
\end{array}\right)_{s}=N\left(\begin{array}{c}
\psi_{1} \\
\psi_{2}
\end{array}\right), \\
N=\left(\begin{array}{cc}
\frac{1}{4 \lambda} & -\frac{1}{2} z_{s} \\
\frac{1}{2} z_{s} & -\frac{1}{4 \lambda}
\end{array}\right) \\
+\sum_{j=1}^{N} \frac{\lambda_{j}}{\lambda^{2}-\lambda_{j}^{2}}\left(\begin{array}{c}
2 \lambda \psi_{1 j} \psi_{2 j} \\
\lambda_{j}\left(\psi_{1 j}^{2}+\psi_{2 j}^{2}\right)-\lambda\left(\psi_{1 j}^{2}-\psi_{2 j}^{2}\right)
\end{array} \begin{array}{c}
-\lambda_{j}\left(\psi_{1 j}^{2}+\psi_{2 j}^{2}\right)-\lambda\left(\psi_{1 j}^{2}-\psi_{2 j}^{2}\right) \\
-2 \lambda \psi_{1 j} \psi_{2 j}
\end{array}\right) .
\end{gathered}
$$

Remark 2. The system (32) is a new integrable deformation of sine-Gordon equation with the Lax pair given by (33). The system (32) can be regarded as a new kind of sine-Gordon equation with self-consistent source which is different from the sine-Gordon equation with self-consistent source in [24].

\section{Solutions to the IDSGE}

\subsection{Multi-soliton solutions}

Introducing the dependent variable transformation

$$
\begin{aligned}
& z=2 i \ln \frac{\bar{f}}{f}, \\
& \psi_{1 j}=i\left(\frac{g_{j}}{f}-\frac{\bar{g}_{j}}{\bar{f}}\right), \quad \psi_{2 j}=-\left(\frac{\bar{g}_{j}}{\bar{f}}+\frac{g_{j}}{f}\right), \quad j=1, \cdots, N
\end{aligned}
$$

the IDSGE (32) can be transformed into the bilinear form

$$
\begin{aligned}
& D_{y} D_{s} f \cdot f=\frac{1}{2}\left(f^{2}-\bar{f}^{2}\right)-8 i \sum_{j=1}^{N} \lambda_{j}{\overline{g_{j}}}^{2}, \\
& D_{y} g_{j} \cdot f=-\lambda_{j} \bar{g}_{j} \bar{f}, \quad j=1,2, \cdots, N,
\end{aligned}
$$


where $\bar{f}$ and $\bar{g}_{j}$ are complex conjugates of $f$ and $g_{j}, D$ is the well-known Hirota bilinear operator defined by 22 ]

$$
D_{y}^{m} D_{s}^{n} f \cdot g=\left.\left(\partial_{y}-\partial_{y^{\prime}}\right)^{m}\left(\partial_{s}-\partial_{s^{\prime}}\right)^{n} f(y, s) g\left(y^{\prime}, s^{\prime}\right)\right|_{y^{\prime}=y, s^{\prime}=s} .
$$

The Wronskian determinant is defined as [25]

$$
W=\left|\Psi^{(0)}, \Psi^{(1)}, \cdots, \Psi^{(N-1)}\right|=|0,1, \cdots, N-1|=|\widehat{N-1}|,
$$

where $\Psi^{(0)}=\Psi=\left(\Psi_{1}(y, s), \Psi_{2}(y, s), \cdots, \Psi_{N}(y, s)\right)^{T}$ and $\Psi^{(j)}=\frac{\partial^{j} \Psi}{\partial y^{j}}$.

Since the bilinear form (35) is same as that in [24] except replacing $g_{j}$ by $\bar{g}_{j}$, we may find the solution of IDSGE (32) by directly using the formulae and notation in [24]. We have the following theorem

Theorem 1. Let

$$
\Psi_{j}=i e^{\xi_{j}}-(-1)^{j} e^{-\xi_{j}}, \quad j=1,2, \cdots, N,
$$

where $\xi_{j}=-\lambda_{j} y-\frac{s}{4 \lambda_{j}}+\alpha_{j}(s), \lambda_{j}$ are real number and we set $\lambda_{1}<\lambda_{2}<\cdots<\lambda_{N}$, then the IDSGE (32) has the Wronskian determinant solutions given by (34) with

$$
\begin{aligned}
& f=|\widehat{N-1}| \\
& g_{h}=(-1)^{h+N} \sqrt{\alpha_{h}^{\prime}(s) \prod_{l=1}^{h-1}\left(\lambda_{h}^{2}-\lambda_{l}^{2}\right) \prod_{l=h+1}^{N}\left(\lambda_{l}^{2}-\lambda_{h}^{2}\right) \overline{\left|\widehat{N-2}, \tau_{h}\right|}} \\
& \tau_{h}=\left(\delta_{h, 1}, \cdots, \delta_{h, N}\right)^{T}, h=1, \cdots, N
\end{aligned}
$$

where $\overline{\left|\widehat{N-2}, \tau_{h}\right|}$ is complex conjugates of $\left|\widehat{N-2}, \tau_{h}\right|$.

The Theorem 1 can be proved in the same way as in [24], we omit it.

When take $N=1$, (34) and (38) give rise to one soliton solution for the IDSGE(32)

$$
\begin{aligned}
& z_{1}=4 \arctan e^{2 \xi_{1}}=2 i \ln \frac{1-i e^{2 \xi_{1}}}{1+i e^{2 \xi_{1}}} \\
& \psi_{11}=\frac{2 \sqrt{\alpha_{1}^{\prime}(s)} e^{3 \xi_{1}}}{1+e^{4 \xi_{1}}}, \psi_{21}=\frac{-2 \sqrt{\alpha_{1}^{\prime}(s)} e^{\xi_{1}}}{1+e^{4 \xi_{1}}} .
\end{aligned}
$$

In Fig1, we plot the single-soliton solution of $z_{1}$ and $\psi_{11}$.

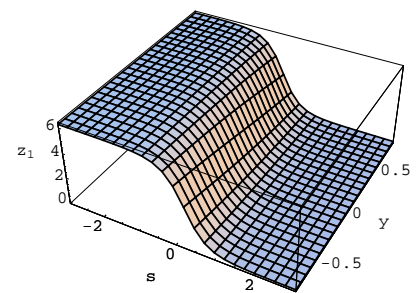

(a)

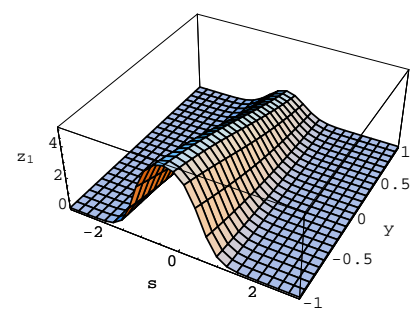

(b)

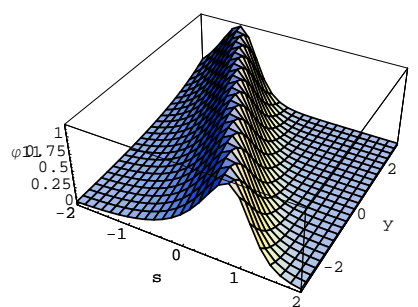

(c)

Figure 1. (a),(b) Single soliton solutions $z_{1}$ when $\lambda_{1}=0.5, \alpha_{1}(s)=s$, and $\lambda_{1}=$ $0.5, \alpha_{1}(s)=s^{2}$, respectively. (c) The eigenfunction $\psi_{11}$ for $\lambda_{1}=0.5, \alpha_{1}(s)=s$. 
Similarly, when take $N=2$ in (38), we have

$$
\begin{aligned}
& f=\left(e^{\xi_{1}+\xi_{2}}+e^{-\left(\xi_{1}+\xi_{2}\right)}\right)\left(\lambda_{2}-\lambda_{1}\right)-i\left(e^{\xi_{1}-\xi_{2}}-e^{\xi_{2}-\xi_{1}}\right)\left(\lambda_{1}+\lambda_{2}\right), \\
& g_{1}=-\sqrt{\alpha_{1}^{\prime}(s)\left(\lambda_{2}^{2}-\lambda_{1}^{2}\right)}\left(e^{-\xi_{2}}+i e^{\xi_{2}}\right), g_{2}=\sqrt{\alpha_{2}^{\prime}(s)\left(\lambda_{2}^{2}-\lambda_{1}^{2}\right)}\left(e^{-\xi_{1}}-i e^{\xi_{1}}\right) .
\end{aligned}
$$

(34) and (401) give to the two soliton solutions for the IDSGE(32). Fig 2. describes the shapes and interactions of two soliton solution for $z_{2}$ and $\psi_{11}$. Also Fig 2. shows the interactions are elastic collisions and the influence on two soliton solution for $z_{2}$ and $\psi_{11}$ for taking different $\alpha_{j}(s)$.

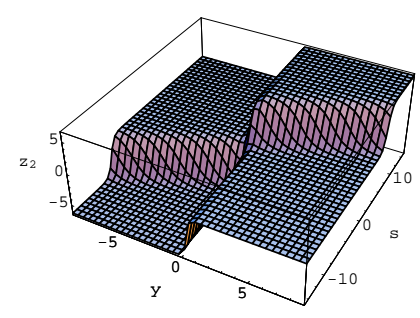

(a)

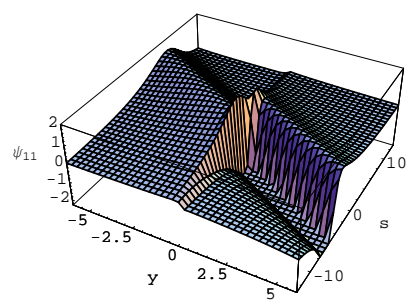

(c)

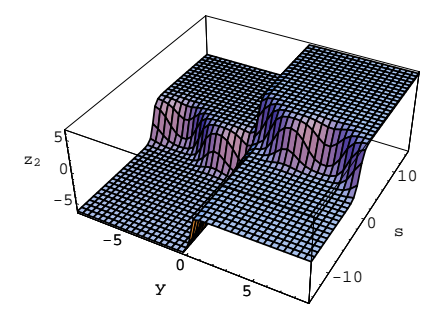

(b)

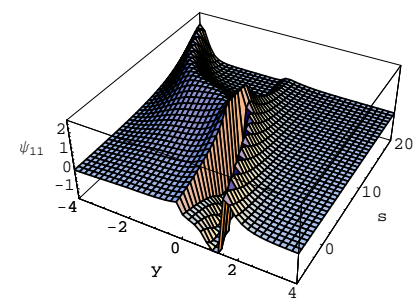

(d)

Figure 2.(a),(b) The shapes and interactions for the two soliton solutions $z_{2}$ when $\lambda_{1}=$ $-0.1, \lambda_{2}=1, \alpha_{1}(s)=s, \alpha_{2}(s)=s$ and $\lambda_{1}=-0.1, \lambda_{2}=1, \alpha_{1}(s)=\sin 2 s, \alpha_{2}(s)=\cos s$, respectively. (c),(d) The shapes and interactions for $\psi_{11}$ when $\lambda_{1}=-0.1, \lambda_{2}=0.2, \alpha_{1}(s)=$ $2 s, \alpha_{2}(s)=s$, and $\lambda_{1}=-0.1, \lambda_{2}=0.2, \alpha_{1}(s)=4 s, \alpha_{2}(s)=\cos s$, respectively.

Notice that solutions (38) contains arbitrary $s$ functions $\alpha_{j}(s)$. This implies that the insertion of non-homogeneous terms into the soliton equation may cause the variation of the speed and shape of soliton. So the dynamics of solutions of IDSGE(32) turns out to be much richer than that of solutions of sine-Gordon equation.

\subsection{Negaton solutions and positon solutions}

For $N=2$, taking $\alpha_{1}(s)=c_{1}, \alpha_{2}(s)=\left(\lambda_{2}-\lambda_{1}\right) e(s)+c_{1}-\frac{1}{2} i \pi$, then (37) leads to

$$
\Psi_{1}=e^{-\xi_{1}}+i e^{\xi_{1}}, \Psi_{2}=-i\left(e^{-\xi_{2}}+i e^{\xi_{2}}\right),
$$


where $\xi_{1}=-\lambda_{1} y-\frac{s}{4 \lambda_{1}}+c_{1}, \xi_{2}=-\lambda_{2} y-\frac{s}{4 \lambda_{2}}+\left(\lambda_{2}-\lambda_{1}\right) e(s)+c_{1}-\frac{1}{2} i \pi, c_{1}$ is a constant and $e(s)$ is a function for $s$. We have

$$
\begin{aligned}
& f=\left|\begin{array}{cc}
\Psi_{1} & \Psi_{1 y} \\
\Psi_{2} & \Psi_{2 y}
\end{array}\right|=\left|\begin{array}{cc}
\Psi_{1} & \Psi_{1 y} \\
\left.\frac{\partial \Psi_{2}}{\partial \lambda_{2}}\right|_{\lambda_{2}=\lambda_{1}} & \left.\frac{\partial^{2} \Psi_{2}}{\partial \lambda_{2} \partial y}\right|_{\lambda_{2}=\lambda_{1}}
\end{array}\right|\left(\lambda_{2}-\lambda_{1}\right)+o\left(\lambda_{2}-\lambda_{1}\right) \\
& =-\left(e^{2 \xi_{1}}+e^{-2 \xi_{1}}+4 i \lambda_{1} \gamma\right)\left(\lambda_{2}-\lambda_{1}\right)+o\left(\lambda_{2}-\lambda_{1}\right) \text {, } \\
& g_{1}=0 \text {, } \\
& g_{2}=\sqrt{\left(\lambda_{2}-\lambda_{1}\right) e^{\prime}(s)\left(\lambda_{2}^{2}-\lambda_{1}^{2}\right)} \overline{\left|\begin{array}{ll}
\Psi_{1} & 0 \\
\Psi_{2} & 1
\end{array}\right|}=\left(\lambda_{2}-\lambda_{1}\right) \sqrt{e^{\prime}(s)\left(\lambda_{2}+\lambda_{1}\right)}\left(e^{-\xi_{1}}-i e^{\xi_{1}}\right),
\end{aligned}
$$

where $\gamma=y-\frac{s}{4 \lambda_{1}^{2}}-e(s)$. Then we obtain the one negaton solution from (34) by taking $\lambda_{2} \rightarrow \lambda_{1}$

$$
\begin{aligned}
& z=2 i \ln \frac{\operatorname{ch} 2 \xi_{1}-2 i \lambda_{1} \gamma}{c h 2 \xi_{1}+2 i \lambda_{1} \gamma} \\
& \psi_{12}=\frac{2 \sqrt{2 \lambda_{1} e^{\prime}(s)}\left(-4 \lambda_{1} \gamma e^{-\xi_{1}}+e^{-\xi_{1}}+e^{3 \xi_{1}}\right)}{\left(e^{2 \xi_{1}}+e^{-2 \xi_{1}}\right)^{2}+16 \lambda_{1}^{2} \gamma^{2}} \\
& \psi_{22}=\frac{2 \sqrt{2 \lambda_{1} e^{\prime}(s)}\left(4 \lambda_{1} \gamma e^{\xi_{1}}+e^{\xi_{1}}+e^{-3 \xi_{1}}\right)}{\left(e^{2 \xi_{1}}+e^{-2 \xi_{1}}\right)^{2}+16 \lambda_{1}^{2} \gamma^{2}} .
\end{aligned}
$$

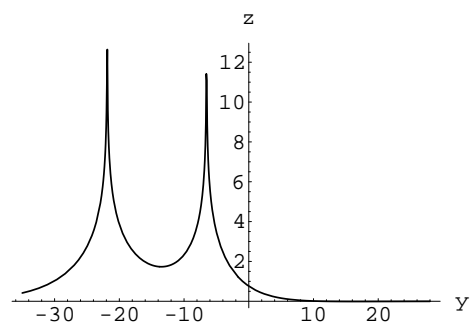

(a)

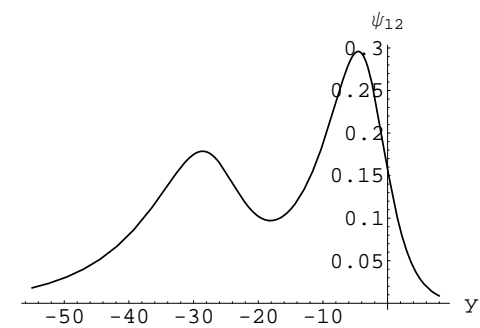

(b)

Figure 3. The shapes for one negaton solution $z$ and $\psi_{12}$ when $\lambda_{1}=0.1, e(s)=2 s, s=0.5$.

The shapes are given in Fig.3. In general, as proposed in [26], the $N$ negaton solution can be obtained from (34), (36) and (38) by replacing $N$ by $2 N$, taking

$$
\begin{aligned}
& \Psi^{(0)}=\left(\Psi_{1},\left.\frac{\partial \Psi_{2}}{\partial \lambda_{2}}\right|_{\lambda_{2}=\lambda_{1}}, \Psi_{3},\left.\frac{\partial \Psi_{4}}{\partial \lambda_{4}}\right|_{\lambda_{4}=\lambda_{3}} \cdots, \Psi_{2 N-1},\left.\frac{\partial \Psi_{2 N}}{\partial \lambda_{2 N}}\right|_{\lambda_{2 N}=\lambda_{2 N-1}}\right)^{T}, \\
& \xi_{2 k-1}=-\lambda_{2 k-1} y-\frac{s}{4 \lambda_{2 k-1}}+c_{2 k-1} \\
& \xi_{2 k}=-\lambda_{2 k} y-\frac{s}{4 \lambda_{2 k}}+\left(\lambda_{2 k}-\lambda_{2 k-1}\right) e_{2 k}(s)+c_{2 k-1}-\frac{1}{2} i \pi
\end{aligned}
$$

and taking $\lambda_{2 k} \rightarrow \lambda_{2 k-1}$. 
In order to derive the positon solution as pointed out in [23], we have to take

$$
\lambda_{1}=i \mu_{1}, \lambda_{2}=i \mu_{2}, c_{1}=-i \bar{c}_{1} .
$$

By similarly calculation, we obtain the following one positon solution

$$
\begin{aligned}
& z=2 i \ln \frac{\cos 2 \eta_{1}+2 \mu_{1} \bar{\gamma}}{\cos 2 \eta_{1}-2 \mu_{1} \bar{\gamma}}, \\
& \psi_{12}=\frac{\sqrt{2 i \mu_{1} e^{\prime}(s)}\left(-4 i \mu_{1} \bar{\gamma} e^{i \eta_{1}}+e^{i \eta_{1}}+e^{-3 i \eta_{1}}\right)}{2\left(\cos ^{2} 2 \eta_{1}-4 \mu_{1}^{2} \bar{\gamma}^{2}\right)}, \\
& \psi_{22}=\frac{\sqrt{2 i \mu_{1} e^{\prime}(s)}\left(4 i \mu_{1} \bar{\gamma} e^{-i \eta_{1}}+e^{-i \eta_{1}}+e^{3 i \eta_{1}}\right)}{2\left(\cos ^{2} 2 \eta_{1}-4 \mu_{1}^{2} \bar{\gamma}^{2}\right)} .
\end{aligned}
$$

where $\bar{\gamma}=y+\frac{s}{4 \mu_{1}^{2}}-e(s), \eta_{1}=\mu_{1} y-\frac{s}{4 \mu_{1}}+\bar{c}_{1}$. Just as the positon solutions of the sineGordon equation are complex [23], one positon solution (46) is also complex and reduces to the positon solution of sine-Gordon equation by taking $e(s)$ to be a constant.

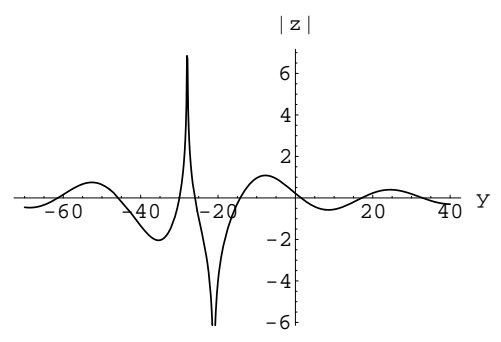

(a)

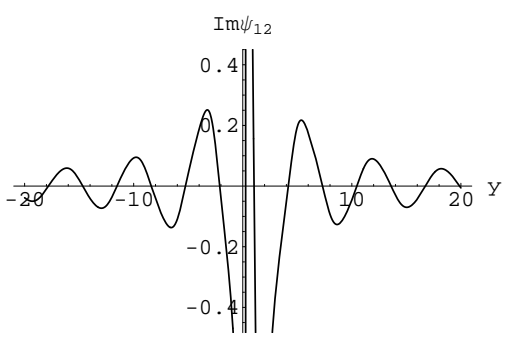

(b)

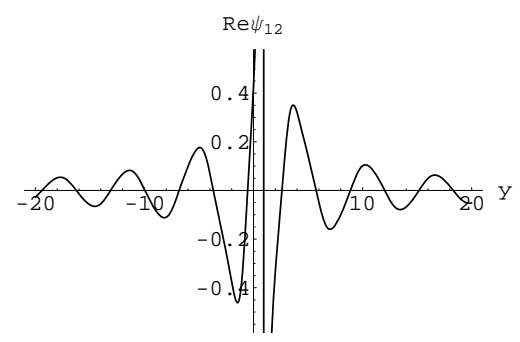

(c)

Figure 4. The shapes for the modulus of one positon solution $z$, the real part and imaginary part of $\psi_{12}$ when $\lambda_{1}=0.1, e(s)=s^{2}, s=1$, respectively.

Fig.4 describes the shapes for the modulus of one positon solution. Similarly, we can find $N$ positon solutions by using (44) and $\lambda_{j}=i \mu_{j}, c_{k}=-i \bar{c}_{k}$ and $\mu_{2 k} \rightarrow \mu_{2 k-1}$.

\section{Solutions for the IDSPE}

\subsection{N-loop soliton solutions}

Proposition 1. Suppose that $z$ and $\psi_{i j},(i=1,2, j=1,2, \cdots, N)$ are solutions of the IDSGE (32), then the solutions of the IDSPE (15) with a parametric representation of $(y, s)$ are given by

$$
\begin{aligned}
& u=z_{s}-2 \sum_{j=1}^{N}\left(\psi_{1 j}^{2}+\psi_{2 j}^{2}\right), \\
& \varphi_{1 j}(x, t)=\psi_{1 j}(y, s), \varphi_{2 j}(x, t)=\psi_{2 j}(y, s), j=1,2, \cdots, N, \\
& x(y, s)=\int \cos z d y=y-\left.2\left(\left.\ln f \bar{f}\right|_{\alpha_{j}(s)=\alpha_{j}}\right)_{s}\right|_{\alpha_{j}=\alpha_{j}(s)},
\end{aligned}
$$


where $\alpha_{j}$ are arbitrary constants.

Proof. It is obviously that (47a) is given by (31). In the following, we prove (47c). From the reciprocal transformation, we have the following linear PDEs for $x$

$$
\frac{\partial x}{\partial y}=\frac{1}{r}, \frac{\partial x}{\partial s}=-\frac{1}{2} u^{2}-2 \sum_{j=1}^{N} \lambda_{j}^{-1} \psi_{1 j} \psi_{2 j} .
$$

By making use of the compatibility of the above two equations, we have

$$
x(y, s)=\int \frac{1}{r} d y=\int \cos z d y .
$$

From (34) and (35), a direct calculation gives

$$
\cos z=1-2(\ln f \bar{f})_{y s}+8 i \sum_{j=1}^{N} \lambda_{j}\left(\frac{g_{j}^{2}}{\bar{f}^{2}}-\frac{\bar{g}_{j}^{2}}{f^{2}}\right) .
$$

When $f$ and $g_{j}$ are given by (37) and (38), the terms with $\alpha^{\prime}(s)$ in $2(\ln f \bar{f})_{y s}$ and the terms $8 i \sum_{j=1}^{N} \lambda_{j}\left(\frac{g_{j}^{2}}{f^{2}}-\frac{\bar{g}_{j}^{2}}{f^{2}}\right)$ are canceled. So the above equation becomes

$$
\cos z=1-\left.2\left(\left.\ln f \bar{f}\right|_{\alpha_{j}(s)=\alpha_{j}}\right)_{y s}\right|_{\alpha_{j}=\alpha_{j}(s)},
$$

namely, for calculating the derivatives with respect to $s$, we regard $\alpha_{j}(s)$ as independent of $s$. Substituting this equation in (49) leads to (47c).

For example, when take $N=1$, by making use of (39) and (47), we obtain the one loop soliton solution for the IDSPE (15)

$$
\begin{aligned}
& u_{1}=\frac{2 e^{2 \xi_{1}}}{\lambda_{1}\left(1+e^{4 \xi_{1}}\right)}, \\
& \varphi_{11}=\frac{2 \sqrt{\alpha_{1}^{\prime}(s)} e^{3 \xi_{1}}}{\left(1+e^{4 \xi_{1}}\right)}, \quad \varphi_{21}=\frac{-2 \sqrt{\alpha_{1}^{\prime}(s)} e^{\xi_{1}}}{\left(1+e^{4 \xi_{1}}\right)} . \\
& x(y, s)=y+\frac{2}{\lambda_{1}\left(1+e^{4 \xi_{1}}\right)} .
\end{aligned}
$$

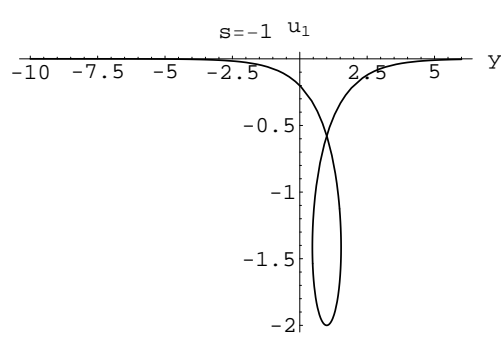

(a)

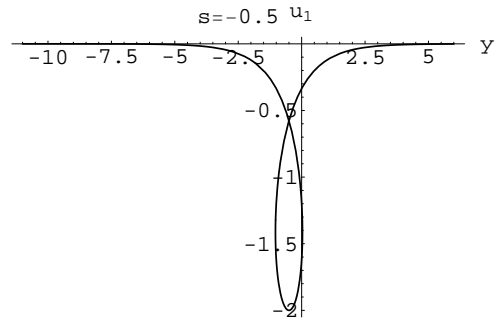

(b)

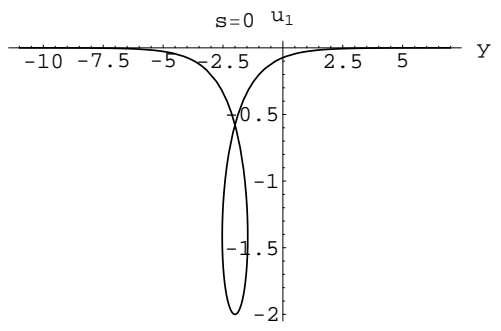

(c) 


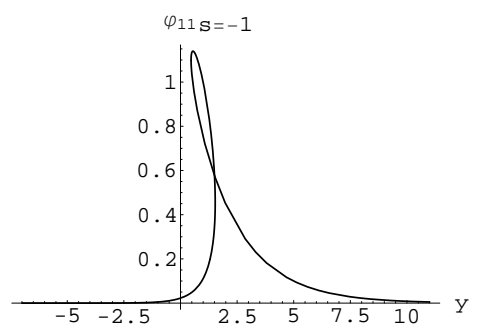

(d)

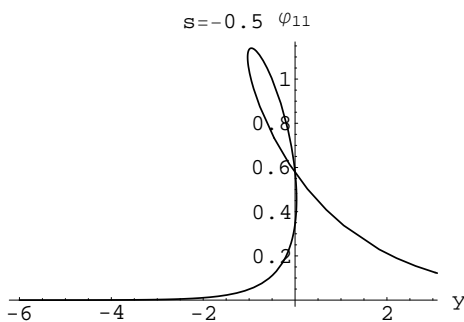

(e)

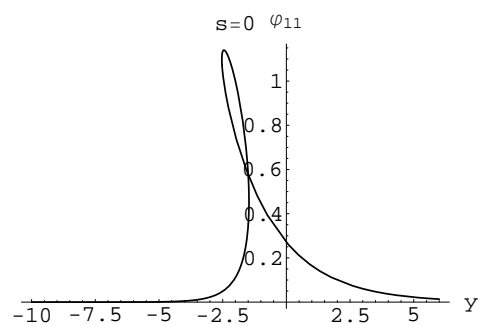

(f)

Figure 5. The shapes and motions for the one loop soliton solutions $u_{1}$ and $\varphi_{11}$ when $\lambda_{1}=-0.5, \alpha_{1}(s)=s$.

Fig.5. shows that the one loop soliton solutions move to the left and keep the shapes. When take $N=2$ in (38), (34) and (47) give to the two loop solution of the IDSPE (15)

$$
\begin{aligned}
u_{2} & =\frac{2\left(\lambda_{1}^{2}-\lambda_{2}^{2}\right)\left[\lambda_{1}\left(1+e^{4 \xi_{1}}\right) e^{2 \xi_{2}}\left(1-12 \lambda_{2} \alpha_{2}^{\prime}(s)\right)-\lambda_{2}\left(1+e^{4 \xi_{2}}\right) e^{2 \xi_{1}}\left(1-12 \lambda_{1} \alpha_{1}^{\prime}(s)\right)\right]}{\lambda_{1} \lambda_{2}\left[\left(1+e^{4 \xi_{1}}\right)\left(1+e^{4 \xi_{2}}\right)\left(\lambda_{1}^{2}+\lambda_{2}^{2}\right)-2 \lambda_{1} \lambda_{2}\left(1-e^{4 \xi_{1}}-e^{4 \xi_{2}}+4 e^{2\left(\xi_{1}+\xi_{2}\right)}+e^{4\left(\xi_{1}+\xi_{2}\right)}\right)\right]} \\
\varphi_{11} & =\frac{4 \sqrt{\left(\lambda_{2}^{2}-\lambda_{1}^{2}\right) \alpha_{1}^{\prime}(s)}\left[\left(1-e^{4 \xi_{2}}+2 e^{2\left(\xi_{1}+\xi_{2}\right.}\right) \lambda_{1}-\left(1+e^{4 \xi_{2}}\right) \lambda_{1}\right] e^{\xi_{1}}}{\left(1+e^{4 \xi_{1}}\right)\left(1+e^{4 \xi_{2}}\right)\left(\lambda_{1}^{2}+\lambda_{2}^{2}\right)-2 \lambda_{1} \lambda_{2}\left(1-e^{4 \xi_{1}}-e^{4 \xi_{2}}+4 e^{2\left(\xi_{1}+\xi_{2}\right)}+e^{4\left(\xi_{1}+\xi_{2}\right)}\right)} \\
\varphi_{21} & =\frac{4 \sqrt{\left(\lambda_{2}^{2}-\lambda_{1}^{2}\right) \alpha_{1}^{\prime}(s)}\left[\left(e^{2 \xi_{2}}-e^{2 \xi_{1}}+e^{2 \xi_{1}+4 \xi_{2}}\right) \lambda_{1}-e^{2 \xi_{1}}\left(1+e^{4 \xi_{2}}\right) \lambda_{2}\right] e^{\xi_{1}}}{\left(1+e^{4 \xi_{1}}\right)\left(1+e^{4 \xi_{2}}\right)\left(\lambda_{1}^{2}+\lambda_{2}^{2}\right)-2 \lambda_{1} \lambda_{2}\left(1-e^{4 \xi_{1}}-e^{4 \xi_{2}}+4 e^{2\left(\xi_{1}+\xi_{2}\right)}+e^{4\left(\xi_{1}+\xi_{2}\right)}\right)} \\
\varphi_{12} & =\frac{4 \sqrt{\left(\lambda_{2}^{2}-\lambda_{1}^{2}\right) \alpha_{2}^{\prime}(s)}\left[\left(2 e^{2 \xi_{1}}-e^{2 \xi_{2}}+e^{4 \xi_{1}+2 \xi_{2}}\right) \lambda_{2}-e^{2 \xi_{2}}\left(1+e^{4 \xi_{1}}\right) \lambda_{1}\right] e^{\xi_{2}}}{\left(1+e^{4 \xi_{1}}\right)\left(1+e^{4 \xi_{2}}\right)\left(\lambda_{1}^{2}+\lambda_{2}^{2}\right)-2 \lambda_{1} \lambda_{2}\left(1-e^{4 \xi_{1}}-e^{4 \xi_{2}}+4 e^{2\left(\xi_{1}+\xi_{2}\right)}+e^{4\left(\xi_{1}+\xi_{2}\right)}\right)} \\
\varphi_{22}= & \frac{4 \sqrt{\left(\lambda_{2}^{2}-\lambda_{1}^{2}\right) \alpha_{2}^{\prime}(s)}\left[\left(1-e^{4 \xi_{1}}+2 e^{2\left(\xi_{1}+\xi_{2}\right)}\right) \lambda_{2}-\left(1+e^{4 \xi_{1}}\right) \lambda_{1}\right] e^{\xi_{2}}}{\left(1+e^{4 \xi_{1}}\right)\left(1+e^{4 \xi_{2}}\right)\left(\lambda_{1}^{2}+\lambda_{2}^{2}\right)-2 \lambda_{1} \lambda_{2}\left(1-e^{4 \xi_{1}}-e^{4 \xi_{2}}+4 e^{2\left(\xi_{1}+\xi_{2}\right)}+e^{4\left(\xi_{1}+\xi_{2}\right)}\right)}
\end{aligned}
$$

Similarly, by making use of (37), (38) and (47), we can obtain the N-loop soliton solution for the IDSPE (15). Fig. 6. describes the shapes and interactions of the two loop soliton solutions and the interactions of two loop soliton solution for $u_{2}$ and $\varphi_{11}$ are shown to be elastic collisions.

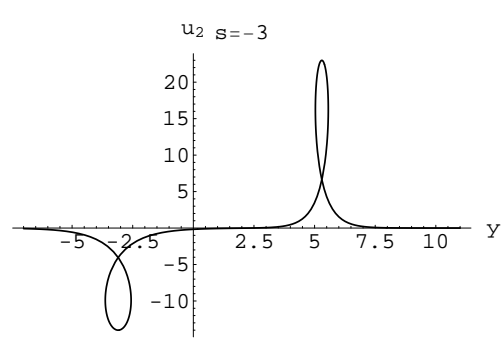

(a)

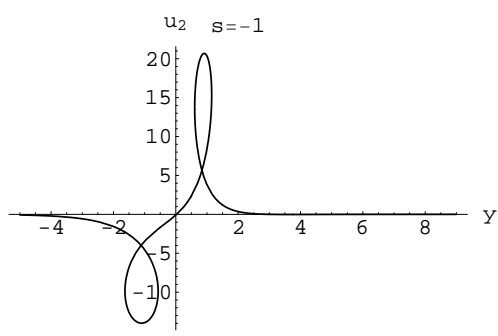

(b)

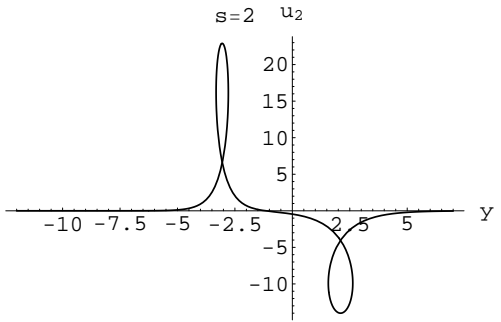

(c) 


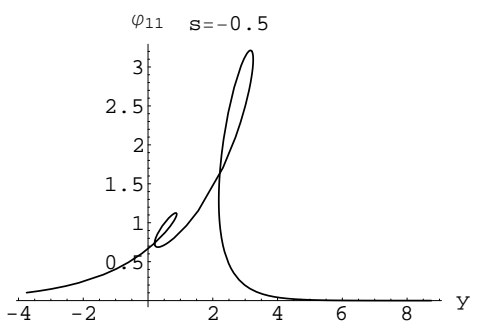

(d)

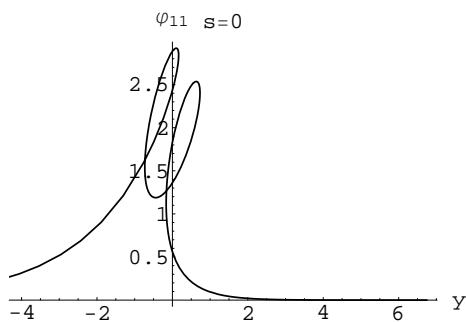

(e)

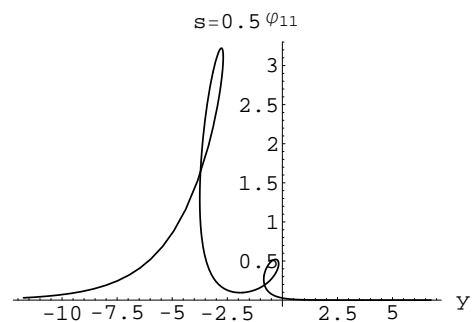

(f)

Figure 6. The shapes and interactions for the two loop soliton solutions $u_{2}$ and $\varphi_{11}$ when $\lambda_{1}=-1, \lambda_{2}=0.5, \alpha_{1}(s)=2 s, \alpha_{2}(s)=s$.

\subsection{Negaton solutions and positon solutions}

By making use of (34), (43) and (47), the one negaton solution for the IDSPE (15) is given

$$
\begin{aligned}
& \begin{array}{l}
u=\frac{-2 e^{\xi_{1}}\left[\left(s-4 \lambda_{1}^{2}(y-e(s))\right)\left(e^{4 \xi_{1}}-1\right)+2 \lambda_{1}\left(1+8 \lambda_{1}^{2} e^{\prime}(s)\right)\left(e^{4 \xi_{1}}+1\right)\right]}{\left[s^{2}+16 \lambda_{1}^{4}(y-e(s))^{2}+2 \lambda_{1}^{2}(1-4 y s)-8 \lambda_{1}^{2} s e(s)\right] e^{4 \xi_{1}}+\lambda_{1}^{2}\left(e^{8 \xi_{1}}+1\right)}, \\
\varphi_{12}=\sqrt{2 \lambda_{1} e^{\prime}(s)} \frac{e^{-\xi_{1}}+e^{3 \xi_{1}}-4 \lambda_{1} \gamma e^{-\xi_{1}}}{2\left(c h^{2} 2 \xi_{1}+4 \lambda_{1}^{2} \gamma^{2}\right)}, \varphi_{22}=\sqrt{2 \lambda_{1} e^{\prime}(s)} \frac{e^{\xi_{1}}+e^{-3 \xi_{1}}+4 \lambda_{1} \gamma e^{\xi_{1}}}{2\left(c h^{2} 2 \xi_{1}+4 \lambda_{1}^{2} \gamma^{2}\right)}, \\
x(y, s)=y-\frac{2\left\{\left[2 s+32 \lambda_{1}^{4}(y+e(s)) e^{\prime}(s)-8 \lambda_{1}^{2}\left(y+e(s)+s e^{\prime}(s)\right)\right] e^{-4 \xi_{1}}+\lambda_{1}\left(e^{-8 \xi_{1}}-1\right)\right\}}{\left[s^{2}+16 \lambda_{1}^{4}(y-e(s))^{2}+2 \lambda_{1}^{2}(1-4 y s)+8 s e(s)\right] e^{-4 \xi_{1}}+\lambda_{1}^{2}\left(e^{-8 \xi_{1}}+1\right)} .
\end{array}
\end{aligned}
$$

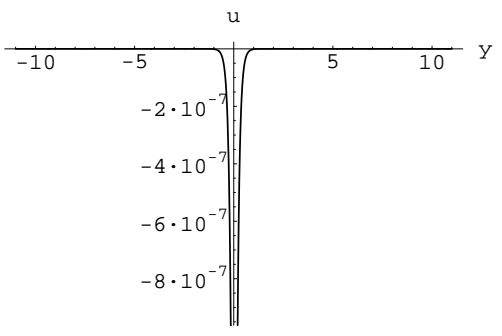

(a)

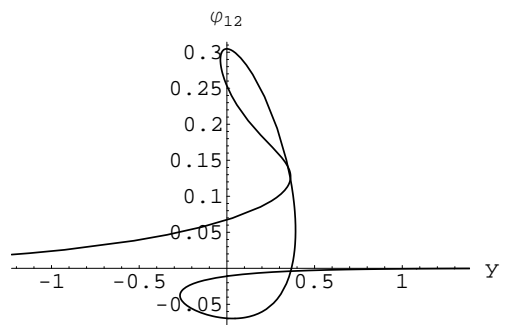

(b)

Figure 7. The shapes for the one negaton solutions when $\lambda_{1}=1, e(s)=s^{3}, s=-0.2$.

The shapes of the one negaton solutions are given in Fig.7. In the same way, by making use of (34), (46) and (47), the one positon solution for the IDSPE (15) is given

$$
\begin{aligned}
& u=\frac{4 i\left[\left(s+4 \mu_{1}^{2}(y-e(s))\right) \sin 2 \eta_{1}-2 \mu_{1}\left(1-8 \mu_{1}^{2} e^{\prime}(s)\right) \cos 2 \eta_{1}\right]}{s^{2}+16 \mu_{1}^{4}(y+e(s))^{2}-2 \mu_{1}^{2}\left(1-4 s y+\cos 4 \eta_{1}-4 s e(s)\right)} \\
& \varphi_{12}=\frac{\sqrt{2 i \mu_{1} e^{\prime}(s)}\left(-4 i \mu_{1} \bar{\gamma} e^{i \eta_{1}}+e^{i \eta_{1}}+e^{-3 i \eta_{1}}\right)}{2\left(\cos ^{2} 2 \eta_{1}-4 \mu_{1}^{2} \bar{\gamma}^{2}\right)} \\
& \varphi_{22}=\frac{\sqrt{2 i \mu_{1} e^{\prime}(s)}\left(4 i \mu_{1} \bar{\gamma} e^{-i \eta_{1}}+e^{-i \eta_{1}}+e^{3 i \eta_{1}}\right)}{2\left(\cos ^{2} 2 \eta_{1}-4 \mu_{1}^{2} \bar{\gamma}^{2}\right)} \\
& x(y, s)=y-\frac{4\left[s+4 \mu_{1}^{2}(y-e(s))-\sin 4 \eta_{1}\right]}{s^{2}+16 \mu_{1}^{4}(y-e(s))^{2}-2 \mu_{1}^{2}\left(1-4 s(y-e(s))+\cos 4 \eta_{1}\right)}
\end{aligned}
$$




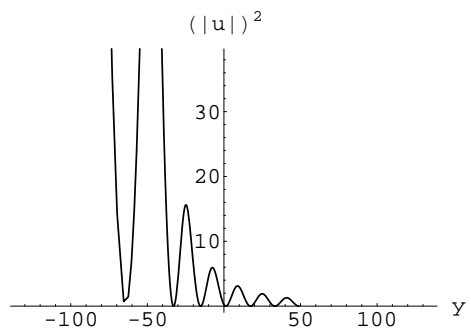

(a)

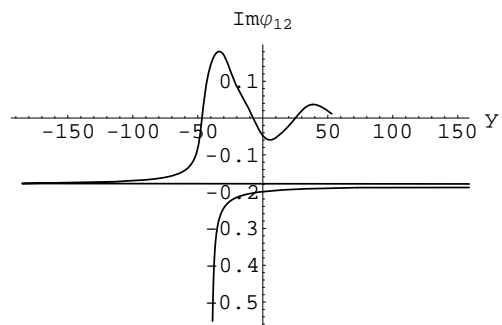

(b)

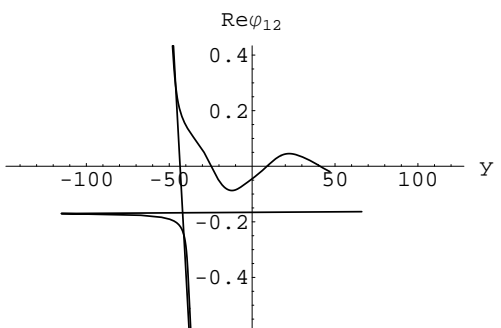

(c)

Figure 8. The shapes for $|u|^{2}$, the real part and imaginary part of $\varphi_{12}$ with $\lambda_{1}=0.1, \quad e(s)=$ $2 s, s=2$, respectively.

The shapes for the one positon solutions are given in Fig.8. By using the N-negaton solutions and N-positon solutions of IDSGE and the inverse reciprocal transformation (47), we can find the N-negaton solutions and N-positon solutions for IDSPE. In the reduced case we can find the new N-negaton solutions and N-positon solutions for short pulse equation by taking all $e_{j}(s)$ to be constants.

\section{Conclusion}

We first derive the integrable deformed short pulse hierarchy and their zero curvature representation. Then we concentrate on the solution of integrable deformed short pulse equation (IDSPE). By proposing a generalized reciprocal transformation, we find a new integrable deformed sine-Gordon equation (IDSGE) and its zero curvature representation. The bilinear equation and the Wronskian determinant solutions for the IDSGE are given. Furthermore, based on the inverse reciprocal transformation and the solutions of the IDSGE, the N-loop soliton solutions, N-negaton and N-positon solutions of the IDSPH are worked out. In the reduced case the new N-negaton solutions and new N-positon solutions for short pulse equation are obtained.

\section{Acknowledgement}

The first author is very grateful to Prof. D. Y. Chen for his help. This work is supported by National Basic Research Program of China (973 Program) (2007CB814800), National Natural Science Foundation of China $(10901090,10801083)$ and Chinese Universities Scientific Fund (2009JS42,2009-2-05)

\section{References}

[1] G. P. Agrawal, Nonlinear Fiber Oplics, 1989, Academic Press, Boston.

[2] T. Schäfer and C. E. Wayne, 2004 Physica D 196 90-105

[3] A. Sakovich, S. Sakovich, On transformations of the Rabelo equations, 2007 SIGMA 3086

[4] M. L. Rabelo, 1989 Stud. Appl. Math., 81 221-248 
[5] A. Sakovich and S. Sakovich, 2005 J. Phys. Soc. Jpn 74 239-241

[6] J. C. Brunelli, 2005 J. Math. Phys. 46123507

[7] J. C. Brunelli, 2006 Phys. Lett. A 353 475-478

[8] B. F. Feng, K. Maruno and Y. Ohta, arXiV: 0912.1914V1

[9] A. Sakovich and S. Sakovich, 2006 J. Phys. A 39 L361-L367.

[10] E. J. Parkes, 2008 Chaos, Solitons and Fractals 38 154-159

[11] V. K. Kuetche, T. B. Bouetou and T. C. Kofane, 2007 J. Phys. Soc. Jpn 76024004

[12] Y. Matsuno, 2007 J. Phys. Soc. Jpn 76084003

[13] Y. Matsuno, 2008 J. Math. Phys. 49073508

[14] V. K. Mel'nikov, 1989 Commun. Math. Phys. 120 451-468, 126 201-215.

[15] J. Leon and A. Latifi, 1990 J. Phys. A 23 1385-1403.

[16] M. Antonowitz and S. R. Wojciechowski, 1992 Phys. Lett. A 165 47-52.

[17] Y. B. Zeng, 1993 J. Phys. A 26 L273-L278 .

[18] Y. B. Zeng, 1994 Physica D 73 171-188.

[19] Y. B. Zeng, W. X. Ma and R. L. Lin, 2000 J. Math. Phys. 41 5453-5489.

[20] Y. B. Zeng, Y. J. Shao, W. M. Xue, 2003 J. Phys. A: Math. Gen. 36 5035-5043.

[21] R. L. Lin, Y. J. Shao, Y. B. Zeng, 2006 Symmetry, Integrability and Geometry: Methods and Application, 2 1-83.

[22] R. Hirota, 1972 J. Phys. Soc. Jpn. 33 1459-1463.

[23] R. Beutler, 1993 J. Math. Phys. 34 3098-3109.

[24] D. J. Zhang and D. Y. Chen, 2003 Physica A 321 467-481.

[25] N. C. Freeman and J. J. C. Nimmo, 1983 Phys. Lett. A 95 1-3.

[26] V. B. Matveev, 2002 Theor. Math. Phys. 131 483-497. 